Research Article

\title{
Engineering the Energy Gap of Cupric Oxide Nanomaterial Using Extreme Learning Machine and Stepwise Regression Algorithms
}

\author{
Abdullah Alqahtani \\ Computer Information System Department, College of Computer Science and Information Technology, Imam Abdulrahman Bin \\ Faisal University, Dammam, Saudi Arabia
}

Correspondence should be addressed to Abdullah Alqahtani; aamqahtani@iau.edu.sa

Received 3 August 2021; Revised 28 September 2021; Accepted 1 October 2021; Published 18 October 2021

Academic Editor: Ahmad A. Adewunmi

Copyright (c) 2021 Abdullah Alqahtani. This is an open access article distributed under the Creative Commons Attribution License, which permits unrestricted use, distribution, and reproduction in any medium, provided the original work is properly cited.

\begin{abstract}
$\mathrm{CuO}$ is a narrow band gap semiconductor with distinct features that render it indispensable in many industrial and technological applications such as environmental friendly catalysts for organic pollutant removal, sensors, photovoltaic, solar cells, batteries, and storage media among others. Engineering of its energy gap becomes imperative and necessary for tailoring its light absorption capacity to a desired level required for a particular application. Elemental doping mechanisms with accompanied lattice distortion symmetry breaking effectively enhance the optical property of this semiconductor and serve as a major route through which material design is achieved. This work develops an extreme learning machine intelligent predictive (ELM-IP) model and stepwise regression (SWR) based model for estimating energy gap of a doped CuO semiconductor. The developed ELM-IP-Sin model which employs sine activation function performs better than the ELM-IP-Sig model (that utilizes sigmoid activation function) and SWR model with a percentage improvement of $14.15 \%$ and $50.05 \%$, respectively, using root mean square error (RMSE) metric, while the developed ELM-IP-Sig model outperforms the SWR-based model. The developed models further investigate the dependence of $\mathrm{CuO}$ energy gap on iron and cobalt impurity incorporation, and the obtained results agree well with measured values. The outstanding performance of the developed models is highly meritorious in tailoring the light response capacity of $\mathrm{CuO}$ semiconductor for photocatalytic and optoelectronics applications at a reduced cost while the experimental stress is circumvented.
\end{abstract}

\section{Introduction}

Copper II oxide $(\mathrm{CuO})$ is a transition metal oxide monoclinic p-type semiconductor with fascinating properties such as nontoxicity, low cost, good stability, narrow energy gap, and natural abundance of its elemental constituents $[1,2]$. The attractive features exhibited by $\mathrm{CuO}$ semiconductor motivate its potentialities in technological and industrial applications such as sensors, solar cell, photocatalysis, supercapacitors, lithium-ion batteries, spintronics, adsorbents, and electrode of photocells among others [3, 4]. Application of this semiconductor for solar energy conversion as well as catalysts attracts significant attention due to its high selectivity and activities in reduction and oxidation reaction [3]. Photodegradation and photocatalytic activities of $\mathrm{CuO}$ semiconductor can be enhanced by band gap engineering through incorporation of suitable foreign materials known as dopants (such as iron and cobalt) with appropriate concentrations [5]. The incorporated dopants frequently stretch and distort the monoclinic crystal structure of $\mathrm{CuO}$ semiconductor and ultimately shrink or widen the energy gap of the semiconductor. Experimental synthesis of crystalline and high-quality $\mathrm{CuO}$ semiconductor with desired energy gap optical property is challenging which necessitates the need for computational technique that can aid material design and enhances tailoring of energy gap to a value desired for specific applications. This work develops the extreme learning machine intelligent predictive (ELM-IP) model and stepwise regression (SWR) based model for determining the energy gap of $\mathrm{CuO}$ semiconductor after dopants incorporation using structural lattice parameters as model descriptors. 
The p-type nature of $\mathrm{CuO}$ semiconductor comes into being due to the presence of acceptor levels attached to vacancies in copper atoms, where charge compensation is achieved through incorporation of impurities or formation of $\mathrm{Cu}^{3+}$ with equivalent concentration [6]. Improvement in its optical properties due to dopants inclusion as well as varying experimental conditions has been reported in the literature [7-9]. Doping introduces crystal defects into $\mathrm{CuO}$ semiconducting crystal structure which serves as points of photon scattering. The optical transition of pure $\mathrm{CuO}$ semiconductor occurs directly from valence to conduction band while $3 \mathrm{~d}$ state doping leads to the creation of surface states within the semiconductor band gap. Oxygen vacancies due to doping are responsible for the modified surface states and redshift behavior that signifies energy gap reduction $[10,11]$. $\mathrm{CuO}$ semiconductor photo-induced behavior can be easily modified by oxygen vacancies which serve as photo-excited trap sites for charge recombination suppression [12]. Foreign material incorporation as well as varying experimental conditions potentially changes the bond length $(\mathrm{Cu}-\mathrm{O})$ and bond angles $(\mathrm{Cu}-\mathrm{O}-\mathrm{Cu})$ of $\mathrm{CuO}$ semiconductor which in turn alters the lattice parameters [13]. The number of oxygen vacancies present in pure and un-doped $\mathrm{CuO}$ lattice is altered when $\mathrm{Cu}^{2+}$ in the $\mathrm{CuO}$ semiconductor is replaced by dopant atoms. This oxygen vacancy variation influences the number of $\mathrm{Cu}-\mathrm{O}$ bond as well as $\mathrm{Cu}-\mathrm{O}-\mathrm{Cu}$ bond angle which subsequently affects the $\mathrm{O}-\mathrm{Cu}-\mathrm{O}$ bond angle of the material. The substitutional sites continue to accommodate the dopant atoms until thermodynamic limit is attained after which interstitial sites are occupied [13]. When dopant atoms are introduced into the $\mathrm{CuO}$ semiconductor, free electrons are released depending on the nature of the dopants. Electron exchange interaction occurs due to the generated free electrons which leads to electron exchange potential that shrink the semiconductor energy gap [13]. Similarly, effective oxidation states differences due to substituting $\mathrm{Cu}^{2+}$ ions with ions of higher oxidation states which leads to electron donation which induces $\mathrm{Cu} \mathrm{s-}$ orbital hybridization that causes energy gap alteration. Extreme learning machine is proposed in this work to approximate nonlinear relationship between energy gap and monoclinic crystal lattice parameters of $\mathrm{CuO}$ semiconductor purposely to develop a robust model through which the energy gap of $\mathrm{CuO}$ semiconductor can be easily controlled to enhance light harvesting capacity of the semiconductor. The stepwise regression- (SWR-) based model is also developed in this work due to ease of implementation of its empirical relations for attaining quick and precise material design procedures that facilitate technological and industrial application of tailored energy gap semiconductors.

Extreme learning machine (ELM) is an extended form of artificial neural network with distinct characteristic features that translate to robust function approximation strength which promotes its wider applicability in solving many real life sciences and engineering problems [14-17]. ELM is characterized with three different layers which include input, hidden, and output layers. The algorithm trains a single hidden neural network with randomized input biases and weights while the output weights are analytically assessed
[18]. It ensures higher efficiency with excellent learning accuracy $[19,20]$. This approach of learning adopted by ELM algorithm gains learning speed and attains reliable generalization capacity as compared to a conventional single hidden neural network. Other merits of extreme learning machine include circumvention of overfitting and ease of convergence to global solution. These unique qualities are utilized in this work in modeling the energy gap of $\mathrm{CuO}$ semiconductor using monoclinic lattice distortion as model descriptors.

The stepwise regression algorithm is a regression model that systematically combines descriptors in accordance to their significance for effective prediction of the desired target $[21,22]$. Its strength for best subset selection conserves computational time while model precision as well as accuracy is further strengthened or maintained [23]. The descriptors that have the highest impact and contribution to the residual sum of squares are given priority while executing addition (forward process) and deletion (backward process) procedures of model development [24-26]. This algorithm is employed in this work to determine the influence of incorporated dopants and distortions along $a$-axis, $b$-axis, and $c$ -axis of $\mathrm{CuO}$ crystal structure on the energy gap. The ease of implementation of stepwise regression generated empirical equations further contributes to its choice in the addressed $\mathrm{CuO}$ energy gap engineering problem.

The remaining part of the manuscript is arranged as follows: Section 2 presents the mathematical formulation of extreme learning machine intelligent predictive algorithm as well as the operational principles of stepwise regression. The computational methodology and strategies of the developed ELM and stepwise regression-based models are presented in Section 3 of the manuscript. The description, sources, and data acquisition strategies are also presented in Section 3. Section 4 presents and discusses the outcomes of the developed ELM and stepwise regression-based models while manuscript conclusions are presented in Section 5.

\section{Mathematical Background of the Employed Intelligent and Stepwise Regression- Based Algorithms}

Mathematical formulation of extreme learning machine is discussed in this section. The section further presents the operational description of stepwise regression algorithm.

2.1. Description of Extreme Learning Machines. Supposing neural network of a single hidden layer is to be developed for approximating nonlinear function connecting lattice parameters of $\eta$ number of $\mathrm{CuO}$ semiconductors (doped with different materials) with their corresponding band gap energy. The dataset from which patterns are to be approximated and acquired is represented by $\left(\gamma_{t}, \xi_{t}\right)$, where input lattice parameters of $t$ doped $\mathrm{CuO}$ semiconductor and band gap energy are, respectively, represented by $\gamma_{t=}$ $\left[\chi_{t 1}, \chi_{t 2}, . . \cdots, \chi_{t j}\right]^{T} \in \mathbb{R}^{j}$ and $\xi_{t}=\left[\xi_{t 1}, \xi_{t 2}, . . \cdots, \xi_{t i}\right]^{T} \in \mathbb{R}^{i}$. Equation (1) expresses network function with $\alpha$ number of 
hidden layer nodes.

$$
\sum_{k=1}^{\alpha} \beta_{k} \phi\left(\omega_{k} \cdot \gamma_{r}+\delta_{k}\right)=\sigma_{r}, \quad r=1,2, \cdots, \eta
$$

where input weights and the activation function are, respectively, represented as $\omega_{k}=\left[\omega_{k, 1}, \omega_{k, 2}, \cdots, \omega_{k, j}\right]^{T}$ and $\phi(\gamma)$. The bias and output weight of $k$ unit of hidden layer are represented as $\delta_{k}$ and $\beta_{k}$, respectively. $\omega_{k} \cdot \gamma_{r}$ stands for dot product between $\omega_{k}$ and $\gamma_{r}$. The algorithm is aimed at minimizing error associated with the predicted band gap of $\mathrm{CuO}$ semiconductor as presented in Equation (2) in such a way that condition contained in Equation (3) regarding $\beta_{k}, \delta_{k}$, and $\omega_{k}$ holds.

$$
\begin{gathered}
\sum_{r=1}^{\eta}\left\|\sigma_{r}-\xi_{r}\right\|=0, \\
\sum_{k=1}^{\alpha} \beta_{k} \phi\left(\omega_{k} \cdot \gamma_{r}+\delta_{k}\right)=\xi_{r}, \quad r=1,2, \cdots, \eta .
\end{gathered}
$$

Equation (4) presents the objective of the ELM algorithm in which $H$ and $\xi^{\text {pred }}$, respectively, represent hidden layer node output and the estimated band gap using the algorithm while $\beta$ stands for output weight.

$$
H \beta=\xi^{\text {pred }} .
$$

The matrix form of Equation (4) is presented in Equation (5) while expression for output weight matrix and estimated band gap of doped $\mathrm{CuO}$ semiconductor are presented in Equations (6) and (7), respectively.

$$
H\left(\omega_{1}, \cdots, \omega_{\alpha}, \delta_{1}, \cdots, \delta_{\alpha}, \gamma_{1}, \cdots, \gamma_{\alpha}\right)=\left[\begin{array}{ccc}
\phi\left(\omega_{1} \cdot \gamma_{1}+\delta_{1}\right) & \ldots & \phi\left(\omega_{\alpha} \cdot \gamma_{1}+\delta_{\alpha}\right) \\
\phi\left(\omega_{1} \cdot \gamma_{\eta}+\delta_{1}\right) & \ldots \phi\left(\omega_{1} \cdot \gamma_{\eta}+\delta_{\alpha}\right)
\end{array}\right]_{\eta X \alpha},
$$

$$
\begin{gathered}
\beta=\left[\begin{array}{c}
\beta_{1}^{T} \\
\vdots \\
\beta_{\alpha}^{T}
\end{array}\right]_{\alpha x i}, \\
\xi^{\text {pred }}=\left[\begin{array}{c}
\xi^{\text {pred }^{T}} \\
1 \\
\vdots \\
\xi^{\operatorname{pred}^{T}} \eta
\end{array}\right]_{\eta x i} .
\end{gathered}
$$

Parameters $\widehat{\omega}_{k}, \widehat{\delta}_{k}$, and $\widehat{\beta}_{k}$ should be obtained in order to train neural network of single hidden layer through Equation (8) in accordance to the operational principle of ELM.

$$
\left\|H\left(\widehat{\omega}_{k}, \widehat{\delta}_{k}\right) \widehat{\beta}_{k}-\xi^{\text {pred }}\right\|=\min _{\omega, \delta, \beta}\left\|H\left(\omega_{k}, \delta_{k}\right) \beta_{k}-\xi^{\text {pred }}\right\| .
$$

Minimization of loss function $Z$ expressed in Equation (9) becomes necessary with the constraint $k=1, \ldots, \alpha$ in order to effectively attain the goal of ELM approximation principle.

$$
Z=\sum_{r}^{\eta}\left(\sum_{k=1}^{\alpha} \beta_{k} \phi\left(\omega_{k} \cdot \gamma_{r}+\delta_{k}\right)-\xi_{r}\right)^{2}
$$

The algorithm uniquely determines the output matrix $H$ of the hidden layer after $\omega_{k}$ and $\delta_{k}$ are randomly obtained. Therefore, the network is conveniently transformed to a linear system $H \beta=\xi^{\text {pred }}$ while $\beta$ is determined through

$$
\widehat{\beta}=\widehat{H} \xi^{\text {pred }}
$$

where $\widehat{H}$ represents the More-Penrose inverse of $H$ matrix

2.2. Stepwise Regression Algorithm. Stepwise regression is a type of multiple linear regression that uses forward-adding and backward-deleting variables to find the best-fitting combination of independent variables for dependent variable prediction [27]. When dealing with a large number of independent factors, determining the appropriate combination of these variables to predict the dependent variable is critical. Stepwise regression can efficiently locate the significant variables from a large amount of raw data based on their special statistical values, allowing the best-fitting model to be built [22]. The stepping technique starts with a model definition and then adds a variable to the preceding model in a stepped forward manner. Although the process is most commonly employed in regression analysis, the basic approach can be used in a variety of model selection situations. Forward selection is all about adding one variable at a time to the subset that has already been selected [24]. For each new variable added or eliminated from the previous model, the forward selection yields the greatest reduction in the residual sum of squares. It can go forward, backward, or both at the same time. Forward selection continues until all variables are incorporated in the model, with no termination constraint [28]. Backward stepwise variable selection begins by selecting subset models from the whole model and deleting each variable whose removal causes the residual sum of squares to grow minimally at each step and continues until only one variable is present in the subset model. The termination rule for stepwise variable selection uses both forward and backward elimination criteria. When all variables in the model meet the criteria to remain and no variables outside the model met the requirement to enter, the variable selection method is brought to stop.

2.3. Mathematical Description of the Employed Performance Measuring Parameters. The feature generalization of the developed models is evaluated using root mean square error (RMSE), correlation coefficient (CC), and mean absolute error (MAE). Equations (11), (12), and (13), respectively, 
depict mathematical formulation of the parameters.

$$
\begin{gathered}
\mathrm{RMSE}=\sqrt{\frac{1}{n} \sum_{i=1}^{n} E r_{i}^{2},} \\
\mathrm{CC}=\frac{\sum\left(\xi_{\mathrm{ex}}-\xi_{\mathrm{ex}}^{*}\right)\left(\xi_{\mathrm{pre}}-\xi_{\mathrm{pre}}^{*}\right)}{\sqrt{\sum\left(\xi_{\mathrm{ex}}-\xi_{\mathrm{ex}}^{*}\right)^{2} \sum\left(\xi a_{\mathrm{pre}}-\xi a_{\mathrm{pre}}^{*}\right)^{2}}}, \\
\mathrm{MAE}=\frac{1}{n} \sum_{i=1}^{n}\left|\mathrm{Er}_{i}\right|,
\end{gathered}
$$

where $\xi_{\text {ex }}$ is the experimental band gap energy, $\xi_{\text {pre }}$ is the predicted band gap energy, $\xi_{\mathrm{ex}}^{*}$ is the mean of experimental band gap energy, $\xi_{\text {pre }}^{*}$ is the mean of predicted band gap energy, Er is the error while $\operatorname{Er}_{i}=\xi_{\mathrm{ex}}-\xi_{\text {pre }}$.

\section{Computational Description and Data Acquisition}

The description of computational details for the developed ELM-IP-Sin, ELM-IP-Sig, and SWR models is presented in this section. Sources as well as description of dataset employed for modeling and simulation are also presented.

3.1. Description of Data Acquisition and Data Source. Modeling of energy gap of doped $\mathrm{CuO}$ semiconductors was carried out using fifty-seven samples of the semiconductor as extracted from the literature [8, 10, 11, 29-37]. The lattice parameters of the semiconducting samples after dopant incorporation serve as the model inputs.

Statistical assessment of the employed dataset from correlation coefficient perspective shows weak degree of linear relationship between the lattice parameters (along $a$-axis, $b$ -axis, and $c$-axis) and the energy gap. Correlation coefficients of $8.96 \%$ as shown in Figure $1(\mathrm{a}), 7.08 \%$ as depicted in Figure $1(\mathrm{~b})$, and $10.6 \%$ as presented in Figure 1(c) were, respectively, obtained between energy gap and $a$-axis lattice parameter, energy gap and $b$-axis lattice parameter, and energy gap and $c$-axis lattice parameter. This is a clear indication that the linear model becomes inadequate for handling this kind of pattern acquisition. Hence, the need for nonlinear modeling tool becomes essential. Doping of $\mathrm{CuO}$ semiconductor results into absorption range blue shift exhibition which influences the semiconductor energy gap due to defect formation in the crystal lattice where photons are scattered [29]. This physically justified relationship between $\mathrm{CuO}$ lattice parameters and the energy gap is well captured through the developed model in this work.

\subsection{Computational Strategies for the Developed Extreme} Learning Machine Intelligent Model. The computational development of ELM model through which band gaps of $\mathrm{CuO}$ semiconductor are estimated took place within MATLAB computing environment. The distorted lattice parameters of the semiconductor which serve as the model inputs and their corresponding energy gaps were initially randomized purposely to ascertain that even distribution of data points within stages of model development is ensured. Subsequently, the dataset was partitioned into training and testing sets in the ratio of $8: 2$. With this partitioning, forty-six of data samples from the semiconductor are employed for model training and weight acquisition while eleven of samples were allotted for model assessment. Computational procedures of the developed ELM-based model are summarized as follows:

(Step A) Hidden node $(\alpha)$ specification and activation function $(\phi(\gamma))$ selection: select hidden nodes from the search space with upper and lower bounds of 100 and 1, respectively. Selection of a function which serves as activation function also falls into this stage of model development. The pool of activation functions includes tribas function, sine function, radbas function, and sigmoid function, among others.

(Step B) Random generation of $\delta_{k}$ and $\omega_{k}$ : generate the bias $\delta_{k}$ and input weights $\omega_{k}$ randomly by implementing initial seed approach within MATLAB computing environment.

(Step C) Matrix computation of the output of hidden layer node $H$ : compute the output of the hidden layer node $H$ through implementation of Equation (5) with the inclusion of the training set of data.

(Step D) Determination of output weight $\beta_{k}$ : calculate the output weight $\beta_{k}$ through Equation (10).

(Step E) Model prediction strength evaluation: determine the prediction strength of the developed ELM-based model by incorporating the testing set of $\mathrm{CuO}$ semiconducting samples into randomly determined biases from Step B, randomized input weights from Step B, and computed output weights from Step D. Root mean square error serves as the parameter for the evaluation.

(Step F) Best model search through various activation function and hidden nodes: repeat Step A to Step E for different activation functions while the number of hidden nodes spans from 1 to 100. Select the model with the lowest root mean square error for any best two activation function. Save the parameters and details of the best models for future implementation. Figure 2 presents the computational flow chat of the developed ELM-based models.

3.3. Computational Method of the Developed Stepwise Regression-Based Model. Development of stepwise regression algorithm with its implementation on $\mathrm{CuO}$ semiconductor energy gap estimation was conducted within MATLAB computing environment. The available dataset extracted from fifty seven doped samples of $\mathrm{CuO}$ semiconductor was 


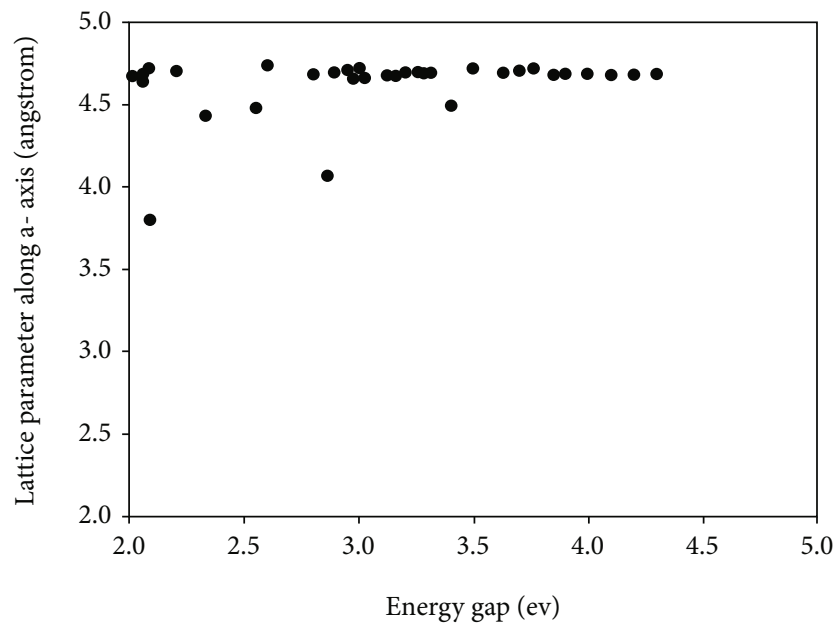

- $\mathrm{CC}=8.96 \%$

(a)

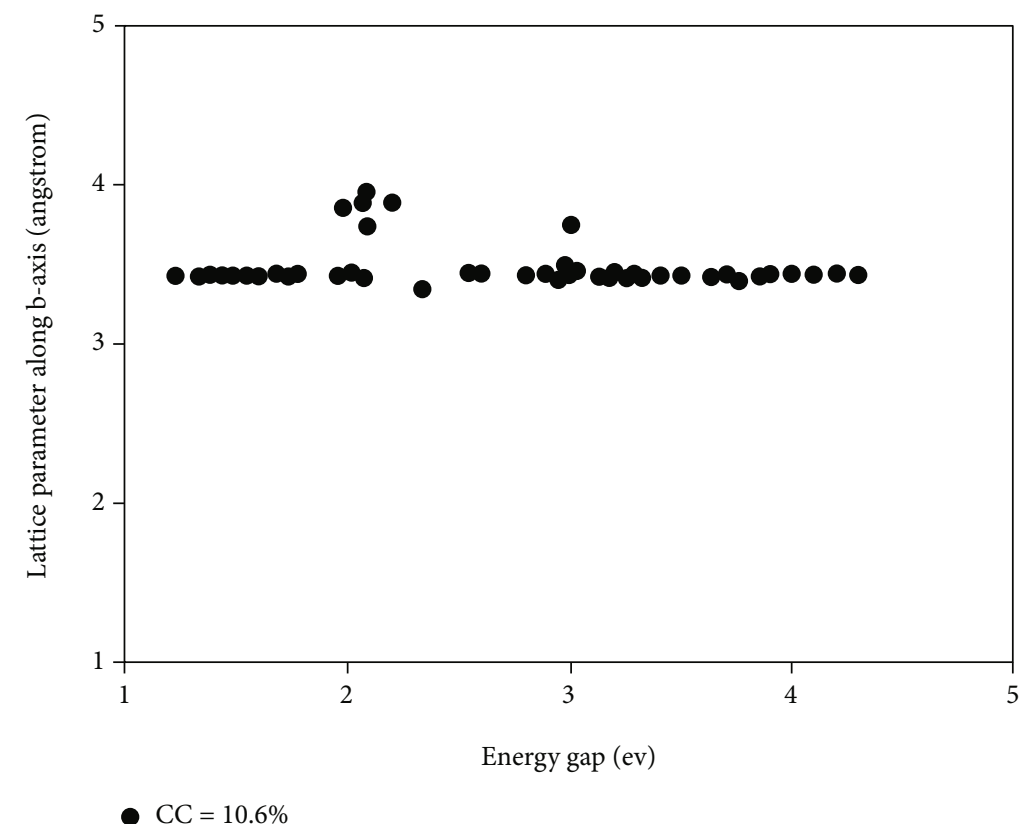

(b)

FIgURe 1: Continued. 


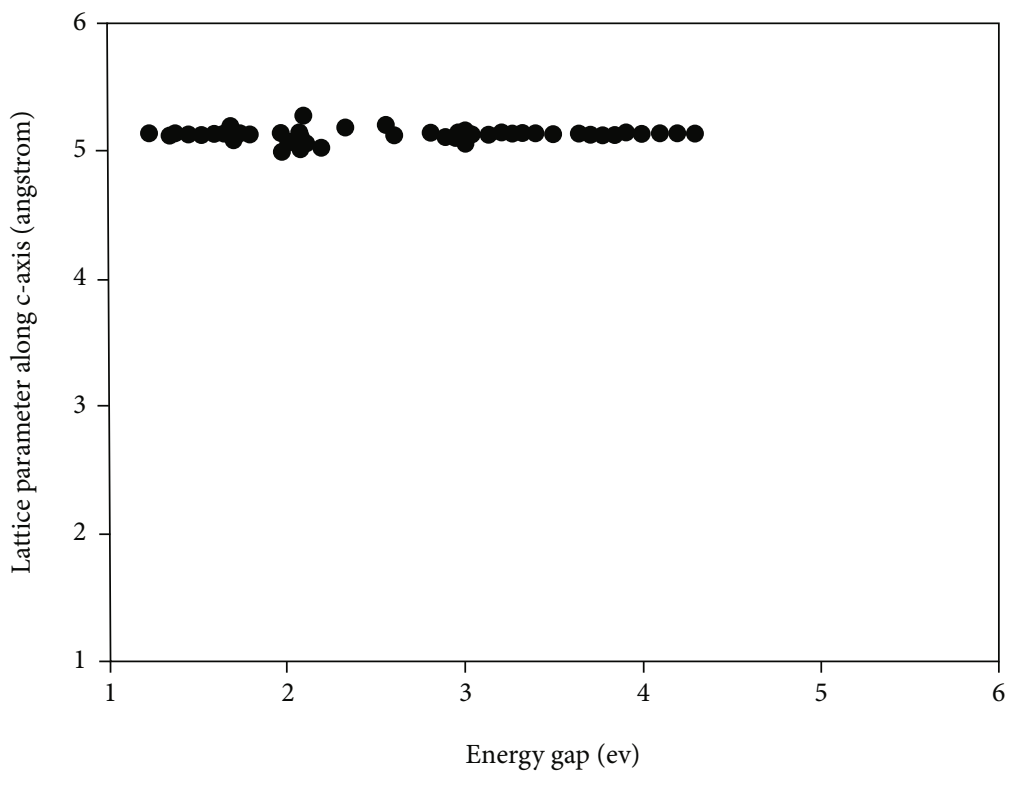

- $\mathrm{CC}=7.08 \%$

(c)

FIgURE 1: Correlation cross-plots between lattice parameters and energy gap of doped CuO semiconductor.

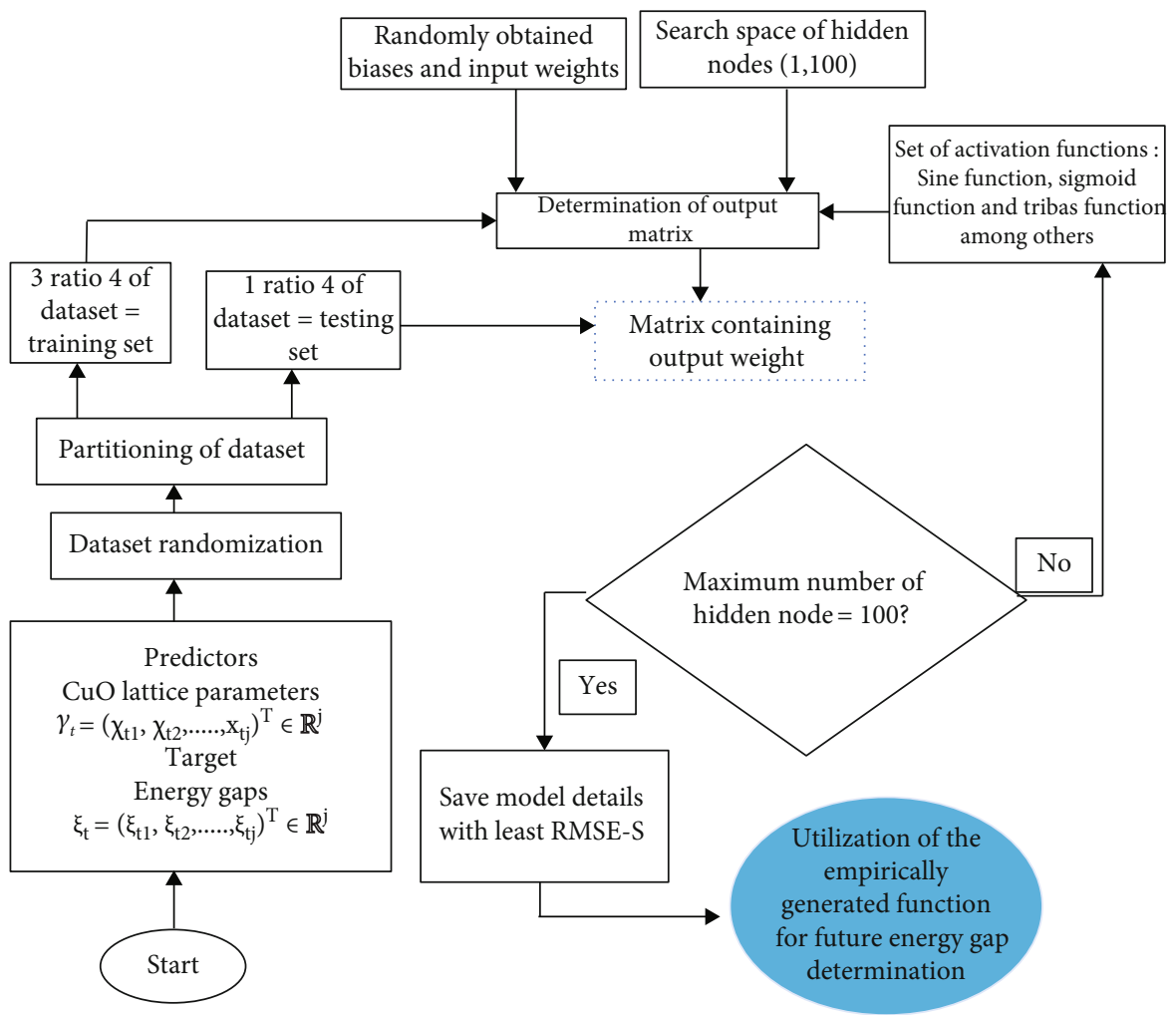

FIGURE 2: Flow chat of the developed ELM-based models.

partitioned into training and testing in the ratio of $4: 1$ while the remaining part of modeling and simulation procedures are summarized as itemized below.
(Step 1) Null model initialization: this step initiates and develops the regression model that is devoid of input descriptors and contains only intercept. 
TABLE 1: ELM-IP-Sin.

\begin{tabular}{cccccc}
\hline$r$ & $\delta_{r}$ & $\lambda_{r}$ & $\psi_{r}$ & $\alpha_{r}$ & $\gamma_{r}$ \\
\hline 1 & -0.0132 & -0.54762 & -0.99652 & 0.723074 & -3973821 \\
2 & -0.26673 & -0.75439 & 0.830837 & 0.500469 & -2477024 \\
3 & -0.5032 & 0.228787 & 0.635176 & 0.353879 & 40239.99 \\
4 & -0.58463 & 0.149019 & -0.69563 & 0.900182 & 30932667 \\
5 & -0.11529 & 0.34942 & -0.92196 & 0.431383 & -6494008 \\
6 & 0.454564 & 0.711009 & 0.811431 & 0.243646 & 4505010 \\
7 & 0.749548 & -0.27287 & -0.96442 & 0.436853 & -5097280 \\
8 & -0.38063 & -0.48935 & 0.967636 & 0.746954 & 10739917 \\
9 & -0.85371 & 0.461603 & -0.04926 & 0.26297 & 53865484 \\
10 & -0.05877 & 0.530997 & -0.89718 & 0.37412 & 47276900 \\
11 & -0.13489 & 0.989218 & 0.702337 & 0.040661 & -8708592 \\
12 & 0.235108 & -0.19435 & -0.27745 & 0.348728 & $-1.6 E+08$ \\
13 & 0.540066 & 0.737888 & 0.48948 & 0.397303 & 9792359 \\
14 & 0.722984 & -0.0377 & 0.221749 & 0.883563 & $-2.3 E+07$ \\
15 & -0.25701 & -0.43914 & -0.66814 & 0.250623 & $-4.3 E+07$ \\
16 & 0.374358 & -0.39904 & 0.493294 & 0.784239 & $-4.5 E+07$ \\
17 & 0.689445 & 0.295375 & -0.57035 & 0.592349 & 10009577 \\
18 & 0.749265 & -0.57982 & -0.85605 & 0.589163 & 4000648 \\
19 & -0.90476 & 0.347213 & -0.8149 & 0.467506 & 12603569 \\
20 & 0.371268 & -0.93299 & 0.890024 & 0.062538 & 12637194 \\
21 & -0.94848 & 0.622763 & 0.999422 & 0.017784 & -2744478 \\
22 & -0.41958 & 0.592968 & -0.29751 & 0.267533 & 21542001 \\
23 & -0.92682 & 0.046075 & 0.250731 & 0.574447 & -6691960 \\
24 & 0.818398 & -0.49557 & 0.96772 & 0.768608 & 6447039 \\
25 & -0.04291 & 0.084512 & 0.574165 & 0.579762 & $1.61 E+08$ \\
26 & -0.57615 & 0.691391 & -0.25016 & 0.878811 & -2432681 \\
27 & 0.627888 & 0.258804 & -0.21725 & 0.516842 & $-1 E+07$ \\
28 & -0.70548 & 0.012369 & -0.82704 & 0.117576 & $-2.3 E+07$ \\
29 & -0.70855 & -0.07151 & -0.30963 & 0.577031 & $-6.9 E+07$ \\
30 & 0.948044 & -0.82533 & -0.02062 & 0.539359 & 15620369 \\
\hline & & & & &
\end{tabular}

(Step 2) Descriptor inclusion: suppose there are $d$ numbers of descriptors for which $k=0,1, ., d, d-k$ linear regression equation is developed for $k=$ 0 using the separated training dataset and null regression model developed in Step 1. This procedure is repeated for other values of $k$ until $d$ number of regression functions is attained.

(Step 3) Model performance characterization through implementation of defined criteria: the generalization performance of each of the developed regression model, as evaluated through testing set of data with root mean square error performance metric, is computer using some defined criteria such as adjusted $R^{2}$, akaike information, sum of squared error, and information criteria.

(Step 4) Substitution of subsequent descriptor into regression model: this step constructs and mod-
TABLE 2: ELM-IP-Sig.

\begin{tabular}{cccccc}
\hline$r$ & $\delta_{r}$ & $\lambda_{r}$ & $\psi_{r}$ & $\alpha_{r}$ & $\gamma_{r}$ \\
\hline 1 & 0.150817 & 0.079697 & 0.177631 & 0.313474 & $-5.3 E+08$ \\
2 & 0.497792 & -0.61757 & 0.639849 & 0.171902 & $8.63 E+08$ \\
3 & 0.485416 & 0.676318 & -0.87409 & 0.892018 & $2.87 E+09$ \\
4 & 0.487825 & -0.32242 & -0.08277 & 0.106813 & $6.12 E+09$ \\
5 & 0.188734 & -0.30055 & -0.54529 & 0.159318 & $3.14 E+10$ \\
6 & 0.546301 & 0.065917 & -0.79026 & 0.584198 & $-1.8 E+09$ \\
7 & 0.013209 & -0.77928 & 0.869907 & 0.851785 & $-5.8 E+08$ \\
8 & 0.459677 & -0.51982 & -0.12869 & 0.833567 & $-1.2 E+09$ \\
9 & 0.613977 & 0.761094 & -0.90338 & 0.111132 & $-2.5 E+09$ \\
10 & 0.544568 & 0.966314 & -0.4342 & 0.513367 & $-1 E+10$ \\
11 & 0.643131 & 0.763693 & -0.49786 & 0.134723 & $8.64 E+09$ \\
12 & -0.73284 & 0.947836 & 0.958558 & 0.603095 & $5.14 E+09$ \\
13 & 0.761244 & 0.444111 & 0.016878 & 0.874886 & $1.77 E+12$ \\
14 & 0.36752 & 0.670529 & 0.904865 & 0.143051 & $-1 E+12$ \\
15 & 0.550825 & -0.77383 & -0.38174 & 0.360877 & $-1.5 E+08$ \\
16 & -0.76514 & -0.25939 & -0.1984 & 0.192199 & $5.02 E+11$ \\
17 & -0.75756 & -0.50261 & 0.207484 & 0.86659 & $2.48 E+10$ \\
18 & -0.29544 & -0.3991 & -0.1797 & 0.116135 & $-9.1 E+10$ \\
19 & -0.13844 & 0.580539 & 0.717628 & 0.775766 & $1.1 E+10$ \\
20 & 0.387409 & -0.47336 & 0.297244 & 0.194758 & $-1.9 E+09$ \\
21 & 0.353658 & -0.41446 & -0.01801 & 0.328061 & $-2.2 E+09$ \\
22 & -0.97888 & 0.060107 & -0.80834 & 0.66384 & $4.02 E+11$ \\
23 & -0.34688 & 0.296413 & 0.95046 & 0.501888 & $3.89 E+10$ \\
24 & 0.658427 & 0.539294 & 0.04276 & 0.048745 & $-8.3 E+11$ \\
25 & -0.88241 & -0.53608 & 0.005874 & 0.464105 & $2.94 E+11$ \\
26 & -0.01034 & 0.088041 & -0.75189 & 0.832069 & $-7.1 E+09$ \\
27 & -0.69636 & -0.5922 & -0.09102 & 0.6647 & $-4.5 E+11$ \\
28 & -0.82263 & -0.11932 & -0.35983 & 0.966932 & $-2.6 E+11$ \\
29 & -0.4391 & -0.04195 & -0.55205 & 0.710365 & $1.08 E+11$ \\
30 & -0.70348 & -0.37291 & 0.717197 & 0.754428 & $8.14 E+08$ \\
31 & -0.52181 & -0.03268 & -0.84714 & 0.40837 & $-2.8 E+11$ \\
\hline & & & & &
\end{tabular}

ifies the regression function obtained in Step 3 through addition of another distortion along other axis. The modified regression function is further validated using testing set of data.

(Step 5) Development of interaction and quadratic terms: there is a construction of interaction terms and pure quadratic of the best regression function with performance evaluation.

(Step 6) Model performance evaluation using root mean square error: the developed $(1+((d(d+1)) / 2))$ regression equations are validated through root mean square error implementation. 
TABLE 3: Performance of the developed intelligent and stepwise regression-based models and their performance superiority.

\begin{tabular}{|c|c|c|c|c|c|c|}
\hline \multirow{2}{*}{ Developed model } & \multicolumn{3}{|c|}{ Training phase } & \multicolumn{3}{|c|}{ Testing phase } \\
\hline & $\mathrm{CC}$ & RMSE & MAE & $\mathrm{CC}$ & RMSE & MAE \\
\hline ELM-IP-Sin & 0.8302 & 0.4985 & 0.3312 & 0.8707 & 0.4761 & 0.3921 \\
\hline ELM-IP-Sig & 0.8417 & 0.4828 & 0.3177 & 0.8382 & 0.5546 & 0.4321 \\
\hline SWR & 0.1471 & 0.8844 & 0.7721 & 0.5930 & 0.9532 & 0.8840 \\
\hline \% improvement of ELM-IP-Sig over ELM-IP-Sin & 1.3661 & 3.1344 & 4.0578 & & & \\
\hline \% improvement of ELM-IP-Sin over ELM-IP-Sig & & & & 3.728839 & 14.1512 & 9.2570 \\
\hline \% improvement of ELM-IP-Sin over SWR & 82.28256 & 43.6399 & 57.1111 & 31.8939 & 50.0519 & 55.6512 \\
\hline \% improvement of ELM-IP-Sig over SWR & 82.52460 & 45.4064 & 58.8515 & 29.2559 & 41.8185 & 51.1270 \\
\hline
\end{tabular}

\section{Results and Discussion}

The empirical relations obtained from extreme learning machine and stepwise regression-based models are presented and discussed in this section. The performance comparison of the developed relations is presented. Energy gap tuning effect of different dopants on $\mathrm{CuO}$ semiconductor is also presented in this section.

4.1. Intelligent and Stepwise Regression-Based Empirical Functions Connecting Lattice Parameters with the Energy Gap. The empirical results of the developed stepwise regression (SWR), extreme learning machine predictive model with sine activation function (ELM-IP-Sin), and extreme learning machine predictive model with sigmoid activation function (ELM-IP-Sig) are, respectively, presented in Equations (11), (12), and (13). The developed SWR model linearly combines the distortion along $a$-axis, $b$-axis, and $c$-axis due to the incorporated dopants while ELM-based models incorporated nonlinearity existing between lattice parameters and the energy gaps.

$$
\begin{aligned}
& \text { SWR }=-33.8206+1.624394 a+0.522291 b+5.256439 c \\
& \text { ELM-IP-Sin }=\sum_{r=1}^{R} \delta_{r} \operatorname{sine}\left(\psi_{r} \cdot a_{r}+\alpha_{r} \cdot b_{r}+\gamma_{r} \cdot c_{r}+\lambda_{r}\right), \\
& \text { ELM-IP-Sig }=\sum_{r=1}^{R} \delta_{r} \operatorname{sigmoid}\left(\psi_{r} \cdot a_{r}+\alpha_{r} \cdot b_{r}+\gamma_{r} \cdot c_{r}+\lambda_{r}\right) .
\end{aligned}
$$

It is expected that the developed SWR-based model performs lower than the ELM-based models since it has been established previously from Figure 1 that the lattice parameters are not linearly correlated with the energy gaps of the distorted $\mathrm{CuO}$ semiconductor. Although the developed SWR model enjoys ease of implementation as compared with the ELM-based model. However, weights that enhance easy implementation of the developed ELM model have been included in Tables 1 and 2. The optimum number of hidden nodes for the developed ELM-IP-Sin and ELM-IP-Sig models is thirty and thirty-one, respectively, as presented in Tables 1 and 2. The performance superiority of the developed intelligent predictive ELM-based models can be attributed to the uniqueness of ELM algorithm to approximate any nonlinear relationship as a result of its strong mathematical background as well as implementation of Moore Penrose inverse matrix. With the provided model weights and biases, the developed ELM models enjoy better performance and ease of implementation as compared with the SWR-based model.

4.2. Performance Evaluation of the Developed Intelligent and Stepwise Regression-Based Models. The performance of the developed extreme learning machine intelligent predictive (ELM-IP) models and stepwise regression (SWR) model is assessed through computation of root mean square error (RMSE), correlation coefficient (CC), and mean absolute error (MAE) during training and testing stages of model development. The results of the model performance are presented in Table 3. Figures 3(a)-3(c), respectively, compare the performance of developed ELM-IP-Sin, ELM-IP-Sig, and SWR models on the bases of CC, MAE, and RMSE metrics during training stage of model development while compari4sons using testing dataset are, respectively, presented in Figures 3(d)-3(f). The developed ELM-IP-Sig model performs better than the ELM-IP-Sin model during training phase with performance improvement of $1.37 \%, 4.06 \%, 4$ and $3.13 \%$ on the basis of CC, MAE, and RMSE, respectively, as presented in Figures 3(a)-3(c) while it outperforms the SWR model during training and testing stage. During the training phase, the developed ELM-IP-Sig outperforms the SWR model with performance superiority of $82.52 \%$, $58.85 \%$, and $45.41 \%$ using CC, MAE, and RMSE metrics while performance enhancements of $29.26 \%, 51.12 \%$, and $41.82 \%$ were, respectively, obtained during the testing stage of model development using the same performance metrics as presented in Figures 3(d)-3(f).

The developed ELM-IP-Sin model outperforms the ELM-IP-Sig model during testing stage with performance improvement of $3.73 \%, 9.26 \%$, and $14.15 \%$ using CC, MAE, and RMSE performance metrics, respectively, while it outperforms the SWR model during the training phase with performance enhancement of $82.28 \%, 57.11 \%$, and 43.64\% using CC, MAE, and RMSE metrics, respectively. While testing the developed ELM-IP-Sin model, the model outperforms the SWR model with performance improvement of $31.89 \%, 55.65 \%$, and 50.05\% using CC, MAE, and RMSE, respectively. The developed ELM-IP-Sin model has demonstrated a high level of performance superiority over 


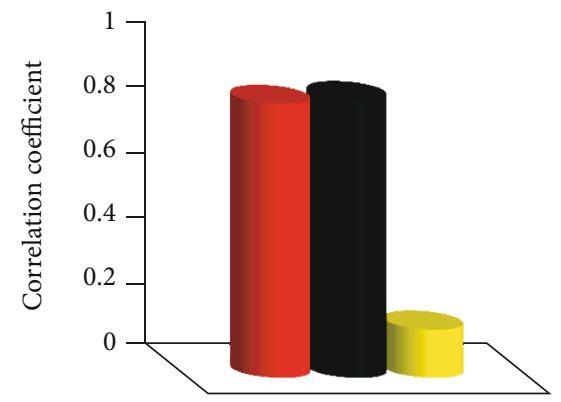

Developed intelligent and stepwise regression based model (training phase)

(a)

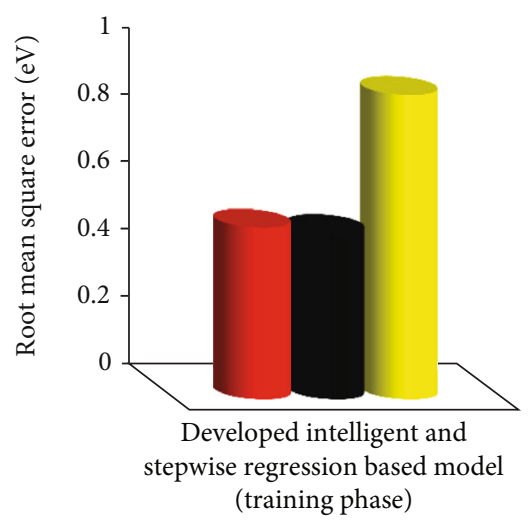

(c)

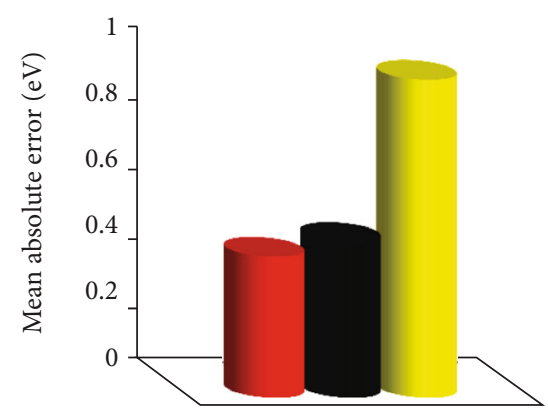

Developed intelligent and stepwise regression based model (testing phase)

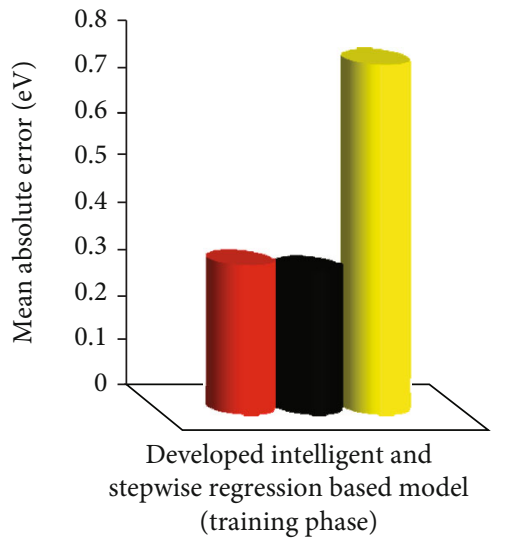

(b)

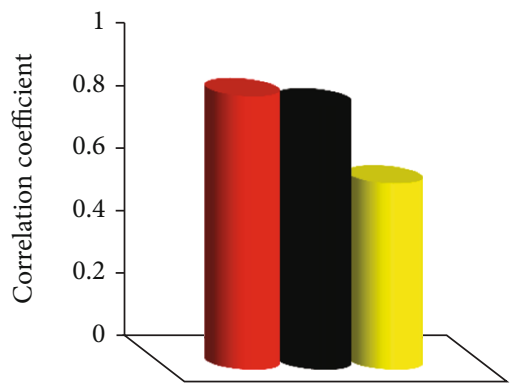

Developed intelligent and stepwise regression based model (testing phase)

(d)

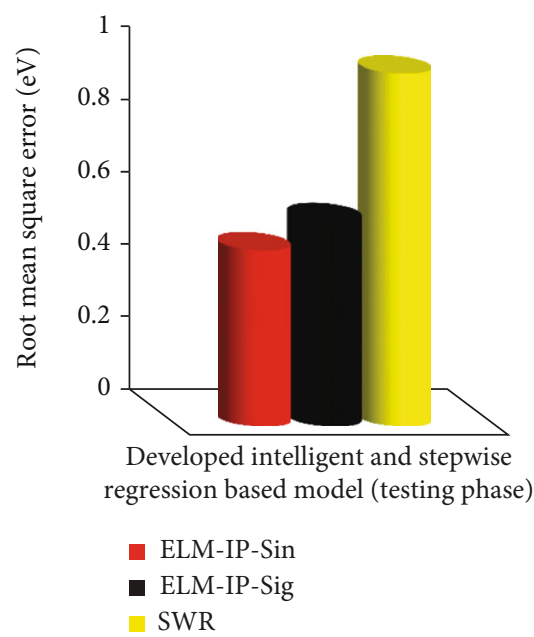

(f)

FIGURE 3: Comparison of performance of the developed intelligent and stepwise regression-based models during training and testing phase.

the SWR and ELM-IP-Sig models during the testing stage of model development.

Table 1 also presents the results of performance superiority of each of the developed model at each stage of model development. Despite the fact that the developed ELM-IPSin model demonstrates superior performance over the SWR and ELM-IP-Sig models, its testing phase shows better performance as compared with the training phase with performance improvement of $4.65 \%, 18.38 \%$, and $4.48 \%$ on the basis of CC, MAE, and RMSE metrics, respectively. The superiority of testing stage over training stage demonstrated by the ELM-IP-Sin model further justifies it robustness, precision, and future potentials to effectively determine the energy gap of doped $\mathrm{CuO}$ semiconductor with high level of accuracy.

4.3. Investigating the Effect of Iron Particle Inclusion on Energy Gap of $\mathrm{CuO}$ Semiconductor Using Developed 


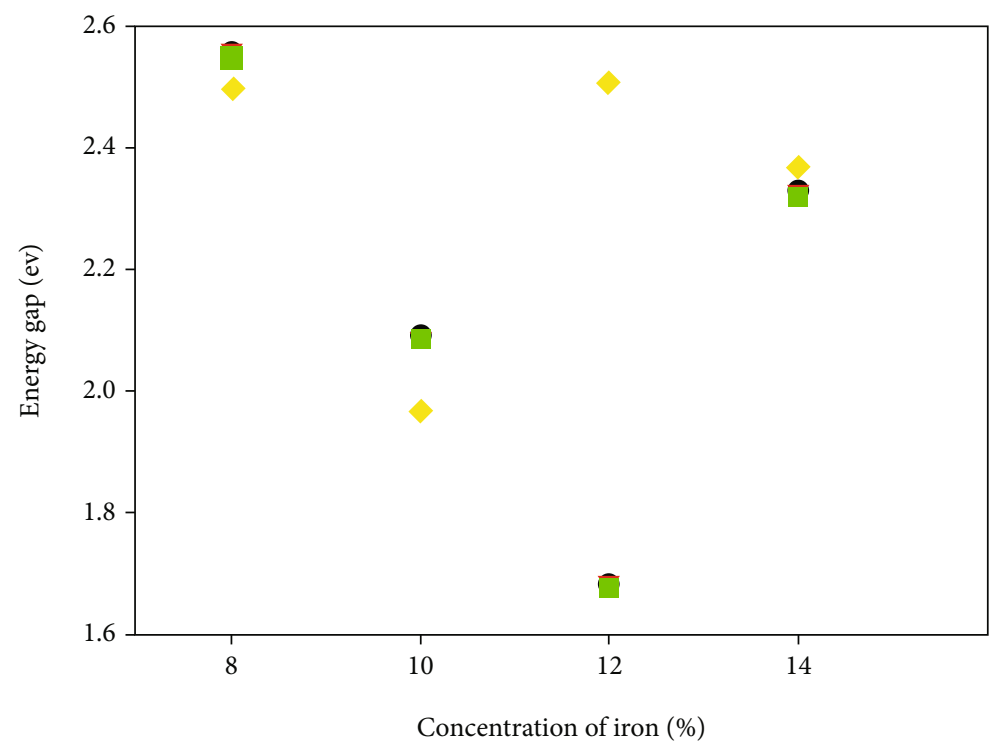
Measured energy gap
ELM-IP-Sin
ELM-IP-Slg
SWR

FIgURE 4: Effect of iron dopants on energy gap of $\mathrm{CuO}$ semiconductor.

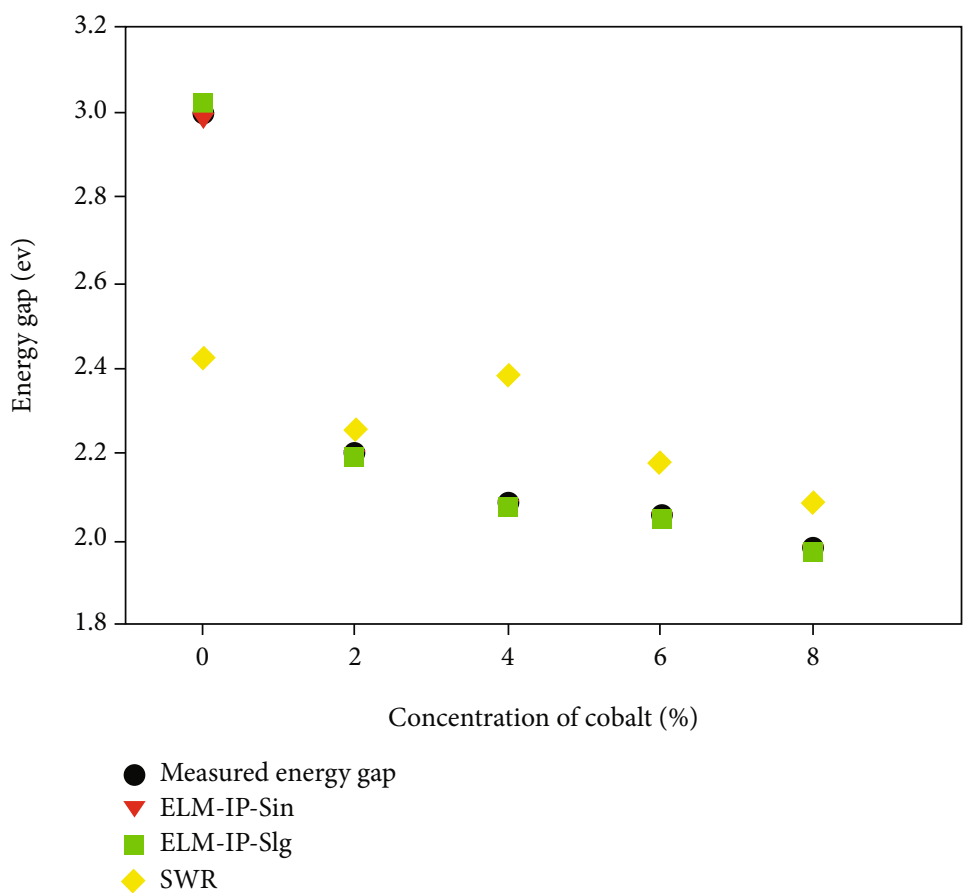

Figure 5: Significant of cobalt particle incorporation on energy gap of doped $\mathrm{CuO}$.

Models. The effect of iron particle inclusion in the lattice structure of $\mathrm{CuO}$ semiconductor using the three developed models is presented in Figure 4 with a comparison to the measured energy gap [4].

The difference between ionic radius of the incorporated iron and the host copper constitutes the observed variation in the unit cell volume which translates to energy gap at dif- ferent concentrations of iron particles. Increase in iron concentration enhances particle agglomeration formation due to improvement in particle small mass, surface tensional force, and surface area/volume ratio. The results of the developed ELM-IP-Sin and ELM-IP-Sig models capture and match the experimental trends excellently well while the estimates of SWR show deviation which can be attributed to 
inadequacy of the SWR algorithm to address nonlinear relationship exhibited by the lattice parameters descriptors and the energy gaps.

\subsection{Effect of Cobalt Foreign Material Inclusion in $\mathrm{CuO}$} Semiconductor Matrix on Energy Gap Using Developed Models. Bang gap engineering effect of cobalt on $\mathrm{CuO}$ semiconductor is investigated by the developed models and presented in Figure 5. The comparison of the model estimates with the measured energy gaps [37] is also presented in the figure. Increase in the concentration of cobalt particles lowers the energy gap of the semiconductor. The observed suppression of energy gap can be attributed to the influence of crystal defects (substitutional and interstitial defects) created by the dopants which enhance photon scattering effect. The doping cobalt ions generate acceptor level (which is shallow) above valence band since atoms of cobalt act as a p-type acceptor which results into energy gap of doped $\mathrm{CuO}$ lower than pure undoped $\mathrm{CuO}$ semiconductor.

The existence of $d-p$ strong coupling between $3 d$ and $2 p$ of oxygen which raises the level of oxygen $2 p$ is also attributed to the observed variation in energy gaps of doped $\mathrm{CuO}$ semiconductor [37]. The estimates of ELM-IP-Sin show consistent closeness with the measured values more than that of ELMIP-Sig model. The developed SWR model performs the least performance with deviation of its estimates from the measured values. The outstanding performance of the developed ELMIP-Sin model justifies the potential of the developed model in adjusting and tuning energy gap of $\mathrm{CuO}$ semiconductor to a level desired for a particular application.

\section{Conclusion}

The energy gap of cupric oxide $(\mathrm{CuO})$ semiconductor with incorporated dopants is modeled using the extreme learning machine intelligent predictive model (ELM-IP) with different activation function (sine and sigmoid) and stepwise regression (SWR) algorithm. The distortions in monoclinic structure of the parent $\mathrm{CuO}$ consequent upon the incorporated dopants, which resulted into crystal defect formation as well as photon scattering, serve as model descriptors. The developed ELM-IP-Sin model outperforms the ELMIP-Sig and SWR models when assessed using correlation coefficient, mean absolute error, and root mean square error metrics. The developed intelligent-based and stepwise regression models investigate the influence of iron and cobalt particle incorporation in $\mathrm{CuO}$ semiconductor matrix on energy gap of the semiconductor and the obtained energy gap agree excellently well with the measured values. The outstanding performance of the developed ELM-IP-Sin model justifies the potential of the model in adjusting and tuning energy gap of $\mathrm{CuO}$ semiconductor to a level desired for a particular technological and industrial application.

\section{Data Availability}

The data employed in building the presented models are contained in the cited sources in Section 3.1 of the manuscript.

\section{Conflicts of Interest}

The author declares that they have no conflicts of interest.

\section{Acknowledgments}

The support of Imam Abdulrahman Bin Faisal University, Dammam, Saudi Arabia, is acknowledged.

\section{References}

[1] S. Bhuvaneshwari and N. Gopalakrishnan, "Enhanced ammonia sensing characteristics of $\mathrm{Cr}$ doped $\mathrm{CuO}$ nanoboats," Journal of Alloys and Compounds, vol. 654, no. 2, pp. 202-208, 2016.

[2] F. Z. Chafi, E. Salmani, L. Bahmad, N. Hassanain, F. Boubker, and A. Mzerd, "First principle calculations with SIC correction of Fe-doped $\mathrm{CuO}$ compound," Computational Condensed Matter, vol. 16, article e00304, 2018.

[3] P. Chand, A. Gaur, A. Kumar, and U. Kumar Gaur, "Structural and optical study of Li doped $\mathrm{CuO}$ thin films on Si $\left(\begin{array}{lll}1 & 0 & 0\end{array}\right)$ substrate deposited by pulsed laser deposition," Applied Surface Science, vol. 307, pp. 280-286, 2014.

[4] K. Kannaki, P. S. Ramesh, and D. Geetha, "Facile hydrothermal synthesis and structural, optical, and morphological investigations on PVP assisted Fe doped $\mathrm{CuO}$ nanocomposites," Materials Today: Proceedings, vol. 3, no. 6, pp. 2329-2338, 2016.

[5] A. Pugazhendhi, S. S. Kumar, M. Manikandan, and M. Saravanan, "Photocatalytic properties and antimicrobial efficacy of Fe doped $\mathrm{CuO}$ nanoparticles against the pathogenic bacteria and fungi," Microbial Pathogenesis, vol. 122, pp. 8489, 2018.

[6] Ş. Baturay, A. Tombak, D. Batibay, and Y. S. Ocak, "N-type conductivity of $\mathrm{CuO}$ thin films by metal doping," Applied Surface Science, vol. 477, pp. 91-95, 2019.

[7] Y. Duan, "Facile preparation of $\mathrm{CuO} / \mathrm{g}-\mathrm{C}_{3} \mathrm{~N}_{4}$ with enhanced photocatalytic degradation of salicylic acid," Materials Research Bulletin, vol. 105, pp. 68-74, 2018.

[8] M. Ponnar, C. Thangamani, P. Monisha, S. S. Gomathi, and K. Pushpanathan, "Influence of Ce doping on $\mathrm{CuO}$ nanoparticles synthesized by microwave irradiation method," Applied Surface Science, vol. 449, pp. 132-143, 2018.

[9] M. Iqbal, A. A. Thebo, A. H. Shah et al., "Influence of Mndoping on the photocatalytic and solar cell efficiency of $\mathrm{CuO}$ nanowires," Inorganic Chemistry Communications, vol. 76, pp. 71-76, 2017.

[10] M. Chaudhary, M. Singh, A. Kumar et al., "Experimental investigation of $\mathrm{Co}$ and $\mathrm{Fe}$-Doped $\mathrm{CuO}$ nanostructured electrode material for remarkable electrochemical performance," Ceramics International, vol. 47, no. 2, pp. 2094-2106, 2021.

[11] A. Sharma, R. K. Dutta, A. Roychowdhury, D. Das, A. Goyal, and A. Kapoor, "Cobalt doped $\mathrm{CuO}$ nanoparticles as a highly efficient heterogeneous catalyst for reduction of 4nitrophenol to 4-aminophenol," Applied Catalysis A: General, vol. 543, pp. 257-265, 2017.

[12] R. Gupta, N. K. R. Eswar, J. M. Modak, and G. Madras, “Ag and $\mathrm{CuO}$ impregnated on $\mathrm{Fe}$ doped $\mathrm{ZnO}$ for bacterial inactivation under visible light," Catalysis Today, vol. 2018, no. 300, pp. 71-80, 2018.

[13] M. R. Islam, J. E. Obaid, M. Saiduzzaman, S. S. Nishat, T. Debnath, and A. Kabir, "Effect of Al doping on the 
structural and optical properties of $\mathrm{CuO}$ nanoparticles prepared by solution combustion method: experiment and DFT investigation," Journal of Physics and Chemistry of Solids, vol. 147, article 109646, 2020.

[14] G. B. Huang, Q. Y. Zhu, and C. K. Siew, "Extreme learning machine: theory and applications," Neurocomputing, vol. 70, no. 1-3, pp. 489-501, 2006.

[15] O. E. Oyeneyin, B. S. Obadawo, A. A. Olanrewaju et al., "Predicting the bioactivity of 2-alkoxycarbonylallyl esters as potential antiproliferative agents against pancreatic cancer (MiaPaCa-2) cell lines: GFA-based QSAR and ELM-based models with molecular docking," Journal of Genetic Engineering and Biotechnology, vol. 19, no. 1, p. 38, 2021.

[16] T. O. Owolabi, M. Amiruddin, and A. Rahman, "Prediction of band gap energy of doped graphitic carbon nitride using genetic algorithm-based support vector regression and extreme learning machine," Symmetry, vol. 13, no. 3, p. 411, 2021.

[17] P. Pi and D. Lima, "Gray level co-occurrence matrix and extreme learning machine for Covid-19 diagnosis," International Journal of Cognitive Computing in Engineering, vol. 2, pp. 93-103, 2021.

[18] D. Gong, W. Hao, L. Gao, Y. Feng, and N. Cui, "Extreme learning machine for reference crop evapotranspiration estimation: Model optimization and spatiotemporal assessment across different climates in China," Computers and Electronics in Agriculture, vol. 187, article 106294, 2021.

[19] R. Hu, E. Ratner, D. Stewart, K. M. Björk, and A. Lendasse, “A modified Lanczos algorithm for fast regularization of extreme learning machines," Neurocomputing, vol. 414, pp. 172-181, 2020.

[20] T. O. Owolabi and M. A. Gondal, "Development of hybrid extreme learning machine based chemo-metrics for precise quantitative analysis of LIBS spectra using internal reference pre-processing method," Analytica Chimica Acta, vol. 1030, pp. 33-41, 2018.

[21] S. M. I. Shamsah and T. O. Owolabi, "Newtonian mechanics based hybrid machine learning method of characterizing energy band gap of doped $\mathrm{ZnO}$ semiconductor," Chinese Journal of Physics, vol. 68, pp. 493-506, 2020.

[22] A. A. Adewumi, M. Ismail, M. A. M. Ariffin et al., "Empirical modelling of the compressive strength of an alkaline activated natural pozzolan and limestone powder mortar," CeramicsSilikáty, vol. 64, no. 4, pp. 407-417, 2020.

[23] B. Pang and Y. Yin, "Development of a dimensionless rodbundle CHF correlation based on stepwise regression method, Part I: determination of the basic form with CHF data obtained with uniform axial heat flux profile," Annals of Nuclear Energy, vol. 143, article 107454, 2020.

[24] M. S. M. Ridzuan, M. Z. Jaafar, and M. M. Zain, "Quantitative structure-activity relationship (QSAR) modelling of $\mathrm{N}$-aryl derivatives as cholinesterase inhibitors," in 2012 IEEE Symposium on Humanities, Science and Engineering Research, pp. 907-912, Kuala Lumpur, Malaysia, 2012.

[25] P. Y. Yang, C. J. Hui, D. J. Tien, A. W. Snowden, G. E. Derfus, and C. F. Opel, "Accurate definition of control strategies using cross validated stepwise regression and Monte Carlo simulation," Journal of Biotechnology, vol. 306, article 100006, 2019.

[26] M. J. Sharma and S. J. Yu, "Stepwise regression data envelopment analysis for variable reduction," Applied Mathematics and Computation, vol. 253, pp. 126-134, 2015.
[27] Y. Chen, R. Shi, S. Shu, and W. Gao, "Ensemble and enhanced $\mathrm{PM}_{10}$ concentration forecast model based on stepwise regression and wavelet analysis," Atmospheric Environment, vol. 74, pp. 346-359, 2013.

[28] L. Jiao and H. Li, "QSPR studies on the aqueous solubility of PCDD/Fs by using artificial neural network combined with stepwise regression," Chemometrics and Intelligent Laboratory Systems, vol. 103, no. 2, pp. 90-95, 2010.

[29] L. Vimala Devi, S. Sellaiyan, T. Selvalakshmi et al., "Synthesis, defect characterization and photocatalytic degradation efficiency of $\mathrm{Tb}$ doped $\mathrm{CuO}$ nanoparticles," Advanced Powder Technology, vol. 28, no. 11, pp. 3026-3038, 2017.

[30] N. M. Basith, J. J. Vijaya, L. J. Kennedy, and M. Bououdina, "Structural, morphological, optical, and magnetic properties of Ni-doped $\mathrm{CuO}$ nanostructures prepared by a rapid microwave combustion method," Materials Science in Semiconductor Processing, vol. 17, pp. 110-118, 2014.

[31] N. Mohamed Basith, J. Judith Vijaya, L. John Kennedy, and M. Bououdina, "Structural, optical and room-temperature ferromagnetic properties of Fe-doped $\mathrm{CuO}$ nanostructures," Physica E: Low-dimensional Systems and Nanostructures, vol. 53, pp. 193-199, 2013.

[32] J. D. Rodney, S. Deepapriya, P. Annie Vinosha et al., "Photofenton degradation of nano-structured $\mathrm{La}$ doped $\mathrm{CuO}$ nanoparticles synthesized by combustion technique," Optik, vol. 161, no. 2, pp. 204-216, 2018.

[33] A. Albert manoharan, R. Chandramohan, R. David prabu et al., "Facile synthesis and characterization of undoped, Mn doped and $\mathrm{Nd}$ co-doped $\mathrm{CuO}$ nanoparticles for optoelectronic and magnetic applications," Journal of Molecular Structure, vol. 1171, pp. 388-395, 2018.

[34] P. Chand, Manisha, and P. Kumar, "Effect of precursors medium on structural, optical and dielectric properties of CuO nanostructures," Optik, vol. 156, pp. 743-753, 2018.

[35] M. Arfan, D. N. Siddiqui, T. Shahid et al., "Tailoring of nanostructures: $\mathrm{Al}$ doped $\mathrm{CuO}$ synthesized by composite-hydroxide- mediated approach," Results Phys, vol. 13, article 102187, 2019.

[36] S. Velliyan and V. Rajendran, "Study on the effect of $\mathrm{Ce}^{3+}$ doping on structural, morphological and optical properties of $\mathrm{CuO}$ nanoparticles synthesized via combustion technique," Physica B: Condensed Matter, vol. 613, article 413015, 2021.

[37] M. M. H. Babu, J. Podder, R. R. Tofa, and L. Ali, "Effect of Co doping in tailoring the crystallite size, surface morphology and optical band gap of $\mathrm{CuO}$ thin films prepared via thermal spray pyrolysis," Surfaces and Interfaces, vol. 25, article 101269, 2021. 